\title{
Thalamic deep brain stimulation for neuropathic pain: efficacy at 3 years' follow-up
}

\author{
*Vasco Abreu, Medical Student, ${ }^{1}$ Rui Vaz, M.D., Ph.D., ${ }^{1,2,3}$ Virgínia Rebelo, M.Psy., ${ }^{4}$ Maria \\ José Rosas, M.D., ${ }^{3,5}$ Clara Chamadoira, M.D., ${ }^{1,2}$ Martin J. Gillies, M.D., ${ }^{6}$ Tipu Z. Aziz, \\ F.Med.Sci. ${ }^{2,6}$ and Erlick A. C. Pereira, D.M., ${ }^{2,7}$
}

${ }^{1}$ Faculdade de Medicina, Universidade do Porto, Portugal; ${ }^{2}$ Departmento de Neurocirugia, Hospital de

São João and Faculdade de Medicina, Universidade do Porto, Portugal; ${ }^{3}$ Center for Neurosciences CUF

Porto, Portugal; ${ }^{4}$ Departamento de Psicologia, Hospital São João, Porto, Portugal; ${ }^{5}$ Departamento de

Neurologia, Hospital São João, Porto, Portugal; and ${ }^{6}$ Department of Neurosurgery and Nuffield

Department of Surgery, Oxford University Hospitals, Oxford, United Kingdom; ${ }^{7}$ Academic Neurosurgery

Unit, St. George's, University of London.

Object. Chronic neuropathic pain is estimated to affect 3\%-4,5\% of the worldwide population, posing a serious burden to society. Deep Brain Stimulation (DBS) is already established for movement disorders and also used to treat some "off-label" conditions. However, DBS for the treatment of chronic, drug refractory, neuropathic pain, has shown variable outcomes with few studies performed in the last decade. Thus, this procedure has consensus approval in parts of Europe but not the USA. This study prospectively evaluated the efficacy at 3 years of DBS for neuropathic pain.

Methods. Sixteen consecutive patients received 36 months post-surgical follow-up in a single-center. Six had phantom limb pain after amputation and ten deafferentation pain after brachial plexus injury, all due to traumas. To evaluate the efficacy of DBS, patient-reported outcome measures were collated before and after surgery, using a visual analog scale (VAS) score, University of Washington Neuropathic Pain Score (UWNPS), Brief Pain Inventory (BPI), and 36-Item Short-Form Health Survey (SF-36).

Results. Contralateral, ventroposterolateral sensory thalamic DBS was performed in sixteen patients with chronic neuropathic pain over 29 months. A postoperative trial of externalized DBS failed in one patient with brachial plexus injury. Fifteen patients proceeded to implantation but one patient with phantom limb pain after amputation was lost for follow-up after 12 months. No surgical complications or stimulation side effects were noted. After 36 months, mean pain relief was sustained, and the median (and interquartile range) of the improvement of VAS score was $52.8 \%$ $(45.4 \%)(\mathrm{p}=0,00021)$, UWNPS was $30.7 \%(49.2 \%)(\mathrm{p}=0,0590)$, BPI was $55.0 \%(32.0 \%)(\mathrm{p}=0,00737)$ and SF-36 was $16.3 \%(30.3 \%)(\mathrm{p}=0,4754)$.

Conclusions. DBS demonstrated efficacy at 3 years for chronic neuropathic pain after traumatic amputation and brachial plexus injury, with benefits sustained across all pain outcomes measures and slightly greater improvement in phantom limb pain.

\footnotetext{
KEYWORDS amputation • brachial plexus injury • Brief Pain Inventory $\quad$ - deep brain stimulation - neuropathic pain • SF-36 • ventral posterolateral thalamus $\quad$ visual analog scale • University of Washington Neuropathic Pain Score
} 


\section{Introduction}

Chronic pain is a considerable burden to society, transcending many other debilitating medical diseases (including cancer, stroke and trauma) ${ }^{1}$ and has been associated with impairment in cognition and attention, ${ }^{2}$ development of psychiatric disorders, ${ }^{3}$ dependence on opioid analgesia and decline in social functioning. ${ }^{4}$ Further, there are some chronic pain states, such as neuropathic pain, which remain difficult or almost impossible to treat with available therapeutics. ${ }^{5}$

Chronic neuropathic pain is estimated to affect $3 \%-4,5 \%$ of the worldwide population, and it is associated with significant loss of productive time, disruption of social life and withdrawal from the workforce. It was recently redefined as pain caused by a lesion or disease of the somatosensory system, ${ }^{6}$ and its symptom severity and duration are often greater than for other types of chronic pain, leading clinicians to consider neurosurgery as a therapeutic option. ${ }^{7}$

At Hospital de São João in Porto, Portugal, there is a pain unit with a large referral area, receiving a great number of patients, many of them refractory to medical treatment. This fact, coupled with our previous experience with DBS for movement disorders since 2002, led us to begin sensory thalamic stimulation for the treatment of neuropathic pain in 2009. These factors together with the available evidence and expert opinion motivated trial of DBS, before recourse to other available surgical techniques. ${ }^{8,9,10}$

Both phantom limb pain after amputation or deafferentation pain after brachial plexus injury fulfil the recently revised definition of neuropathic pain previously mentioned. Phantom limb pain could be considered an uncommon kind of pain with an incidence of 1-2 cases per 100,000 individuals, but has a high prevalence among amputees, affecting $85 \%$ of them. ${ }^{11}$ Usually, stump pain occurs during the first month following amputation, and without treatment, it disappears after 2 or 3 years, except in cases in which phantom pain develops. Despite the pathophysiological mechanisms of this pain being not clearly defined, there are numerous treatments for phantom limb pain described in scientific literature with little clinical evidence supporting any particular therapy. ${ }^{12}$

Brachial plexus injury commonly results in early deafferentation pain, with $25 \%$ of the sufferers experiencing severe neuropathic pain years later, usually refractory to pharmacotherapy. ${ }^{13}$ Brachial plexus injury pains have been dichotomized into either continuous, often burning or throbbing pain and likely involving thalamic neuroplasticity, or shooting paroxysms associated with dorsal horn hyperactivity. ${ }^{14,15}$

Deep brain stimulation (DBS) is established for Parkinson disease, dystonia and other movement disorders. It is also used to treat "off-label" clinical indications such as epilepsy, obsessive-compulsive and psychiatric disorders, Tourette syndrome and cluster headache. ${ }^{16}$ DBS has been performed for more than half a century for chronic pain amelioration. ${ }^{17}$ There is no consensus about the best target for DBS: the ventroposterolateral nucleus (VPL) of the sensory thalamus has been targeted successfully for 4 decades, ${ }^{18}$ as has the periaqueductal and periventricular gray matter. ${ }^{19}$ Evidence supporting the ventroposterolateral nucleus of the sensory thalamus (VPL) as a target for limb pain came from ablative surgery ${ }^{20}$ with considerable experience gathered over the last two decades from the Oxford group drawn upon to start the Porto department. ${ }^{12}$ 
In this paper, we describe a prospective, open-label, consecutive case series study of patients receiving VPL DBS for chronic neuropathic pain after amputation or brachial plexus injury. These patients were treated at a single Portuguese center by an experienced pain unit with a multidisciplinary functional neurosurgery team. The results of our last work, ${ }^{21}$ after one-year follow-up, have shown that DBS demonstrated efficacy at one year for chronic neuropathic pain after traumatic amputation and brachial plexus injury. Thus, our main objective is to understand whether this effectiveness and the improvements in patient-reported outcome measures are maintained after three years or if there is a loss of efficacy, concomitant with development of a tolerance phenomenon, with time.

\section{Methods}

\section{Study Population}

Patients with chronic neuropathic pain were referred nationally by Portuguese pain units to a single-center multidisciplinary team consisting of neurologists, neurosurgeons, neuropsychologists and pain specialists at Hospital de S. João in Porto, Portugal. Selection criteria included neuropathic pain refractory to all medical treatment for at last 2 years, together with the absence of surgical contraindications such as coagulopathy or ventriculomegaly. Patients with psychiatric disorders were excluded by formal psychiatric and psychological evaluation. The study received local ethical approval. Informed consent was obtained.

These criteria resulted in the selection of sixteen patients (Table 1): fourteen of them were male $(87.5 \%)$ and two female (12.5\%). The average age of the study population was $53 \pm 9.1$ years old.

Surgeries were performed between January 2009 and May 2012. The mean duration of symptoms before surgery was $20.2 \pm 12.6$ years. All patients had chronic neuropathic pain caused by traumatic injuries. One patient did not report any pain relief during the trial period and was not fully implanted. Thus, from the original sample of sixteen patients, only fifteen proceeded to full DBS implantation after successful intraoperative and postoperative trials. One patient with phantom limb pain after amputation was lost to follow-up after 12 months (at which point they had a good clinical outcome), leaving fourteen patients with a 36 month follow-up.

\section{Deep Brain Stimulation Procedure}

Under local anesthesia, a Leksell stereotactic frame (Elekta Instruments, Stockholm, Sweden) was applied to the patient's shaved head. A stereotactic head CT scan with a 2-mm slice thickness was fused with preoperative T1- and T2 -weighted MR images of 2-mm slice thickness from a 1.5-T scanner using FrameLink software (Medtronic, Minneapolis, USA). The Leksell stereotactic arc was then fixed to its frame on the awake patient in the semi-sitting position.

The contralateral ventroposterolateral nucleus of the sensory thalamus was targeted for limb pain and found 10-13 mm lateral to the posterior commissure and the effects of macro stimulation assessed 
from $2 \mathrm{~mm}$ above to $5 \mathrm{~mm}$ below the calculated target to elicit paraesthesia or analgesia in the area of pain. No microelectrode recordings were made. The PVG was not explored in this case series due to paucity of evidence that it might be better than the thalamus and the Oxford group's experience that VPL is more easily and safety targeted, being larger and less deep.

Final electrode position was determined by intraoperative clinical assessment that relied upon subjective reporting by the awake patient of paresthesias at pain areas, after stimulation of $5-50 \mathrm{~Hz}$, with a pulse width of $200-450 \mu$ s and amplitude of $0.5-5 \mathrm{~V}$, the wide range of parameters being accounted for by the great individual variation in patient response seen intraoperatively in DBS for pain. Once satisfactory targeting had been achieved by microdrive adjustment, a Medtronic Model 3387 quadripolar electrode was secured in place with a Stimloc skull fixation device (Medtronic). Electrode leads were externalized. Typical surgery duration was $2-3$ hours, including stereotactic planning.

Bedside DBS programming was undertaken during the postoperative period both morning and afternoon, in sessions lasting approximately 30 minutes per patient. Different frequencies from 5 to $50 \mathrm{~Hz}$ were used alongside varied bipolar stimulation over different contacts at increasing pulse widths and amplitudes to optimize analgesia and minimize unpleasant sensations in the painful body part.

After 48 hours of postoperative clinical assessment, a decision was made whether to permanently implant the electrodes under general anaesthesia. The electrodes were connected to a pulse generator (Medtronic Kinetra or Activa PC) implanted in the chest via new extension leads.

Patients ideally left the hospital a day after implantation of the pulse generator. Continuous rather than on-demand stimulation was encouraged, but in addition to the ability to switch the DBS on and off at will, patients were usually given control over its voltage only, which was typically limited by the clinician to a maximum amplitude of $4 \mathrm{~V}$.

\section{Outcome Assessment}

Quantitative assessment of pain and health-related quality of life were performed 1 month before surgery and postoperatively at 1, 3, 6, 12 months, and then annually by independent, blinded assessors trained in pain medicine. They were not involved in caring for the patient and unaware of the details of the neurosurgical treatment given. The Visual Analog Scale (VAS) to rate pain intensity, the University of Washington Neuropathic Pain Score (UWNPS), and Brief Pain Inventory (BPI) were used. ${ }^{22,23}$

A 36-Item Short-Form HealthSurvey (SF-36) on quality of life along with the pain questionnaires were also completed by patients. The SF-36 responses were regrouped into 8 domains of physical functioning: role (physical), bodily pain, general health, vitality, social functioning, role (emotional), and mental health. Results were scored by online tools (http://www.sf-36.org/demos/sf36.html). Norm-based scores allowed comparison between studies.

\section{Statistical Analysis}


Data was first normalised by dividing the test score at each time point by the preoperative score for that test. A Kruskal Wallis test (Non-parametric one-way ANOVA) was then performed on the normalised data for each test score of interest. The null hypothesis of the Kruskal Wallis test was that the group median of the tested score at each time point came from the same distribution. In tests where $\mathrm{p}$ was less than 0.05 , multiple comparison tests using a Tukey-Kramer method were performed to compare individual group medians at each time point to assess which scores at which time points differed significantly from the preoperative score, with a $\mathrm{p}$-value $<0.05$ considered statistically significant and $\mathrm{p}<$ 0.01 considered highly significant. All data is presented as median + interquartile range. 


\section{Results}

Table 1 lists the demographic characteristics of sixteen patients. Neither surgical complications nor side effects from stimulation emerged in this case series. There was also no need for electrode or implantable pulse generator revisions in the post-operative period up to three years. All patients received continuous bipolar DBS with patient control of amplitude up to a clinician-set maximum.

Table 2 lists the improvements of patients who proceeded to full DBS implantation after successful intraoperative and postoperative trials, at different time points: preoperative and 1,2 and 3 years follow-up.

Figure 1 represents the bar graphs showing overall baseline scores and postoperative improvements in the outcome across the entire cohort. As we reported in our paper in 2013 (results after 1 year follow-up), VAS, UW-NPS and BPI scores have improved with statistical significance after 1 month (only SF-36 has improved without it). After 12 months, the median + (interquartile range) of VAS score improvement was $57.8 \%$ (55\%) (p < 0.000098), of UWNPS was 28.7\% (93.0\%) (p=0,0577), of BPI was $49.6 \%(68.5 \%)(\mathbf{p = 0 , 0 0 7 5})$ and of SF-36 was 10.5\% (52.3\%) (p=0.666). Throughout the third year, mean pain relief was sustained, and at 36 months the median of the improvement of VAS score from before surgery was $52.8 \%(45.4 \%)(\mathbf{p}=\mathbf{0 , 0 0 0 2 1})$, of UWNPS was 30.7\% (49.2\%) (p=0,0590), of BPI was $55.0 \%$ $(32.0 \%)(\mathbf{p = 0 , 0 0 7 3 7})$ and of SF-36 was $16.3 \%(30.3 \%)(\mathrm{p}=0,4754)$.

In our paper published in $2013,{ }^{21}$ a comparison of results was made between brachial plexus injury and phantom limb subgroups, reporting that, although both subgroups showed significant improvements initially with DBS, after 12 months amputation pain improved more, with benefits sustained across all pain outcome measures. This tendency continues to be present even after 36 months (Table 2, Figures 2 and 3): among the brachial plexus injury patients, VAS score improved by $40 \%$ $(31,9 \%)(\mathbf{p}=\mathbf{0 . 0 1 2 9 8})$, UWNPS by $22.7 \%$ (37.1\%) $(\mathrm{p}=0.4632)$, BPI by $47.8 \%(62.8 \%)(\mathrm{p}=0.189)$ and SF36 by $16.0 \%(42.7 \%)(\mathrm{p}=0.9953)$. In the amputation group, after 36 months median VAS score improved by $66.7 \%(51.7 \%)(\mathbf{p = 0 . 0 4 9 4})$, UWNPS by $50.8 \%$ (62.9\%) (p=0.3225), BPI by $65.2 \%(31.6 \%)$ $(\mathrm{p}=0.1623)$ and SF-36 by $16.7 \%(140,2 \%)(\mathrm{p}=0.2406)$.

Regarding the UWNPS subscores, highly significant improvements $(\mathrm{p}<0.001)$ were observed in most domains, including intensity, sharp, hot and unpleasant pain. Improvements in dull and deep pain were statistically significant $(\mathrm{p}<0,05)$, but not in cold, sensitive, itchy and superficial pain. To avoid Type I errors, statistical analyses of these subscores by etiological subgroup, was not performed. However, the considerable reductions in hot, sharp, deep and dull UWNPS subscores in both subgroups suggest, as we also reported after one year, that DBS relieved both the paroxysmal and continuous components of neuropathic pain.

Both severity and interference domains of BPI score were improved overall with statistical significance $(p<0,05)$. Moreover, no significant statistical differences were found in each subgroup, perhaps because of the small number of patients in each one.

Analyses of SF-36 subscores across the entire cohort demonstrated significant and sustained improvements in physical functioning $(\mathrm{p}=0,0451)$, social functioning $(\mathrm{p}=0,0093)$ and bodily pain $(\mathrm{p}=0,0052)$, with improvements in general health and vitality also approaching statistical significance. 
Nevertheless, physical role, mental health and emotional role were not significantly improved.

Patient reported outcome measure improvements were clear after 3 years in this cohort. To evaluate the development of tolerance, we compared all outcome scores at 36 months with the previous annual assessments. The difference between VAS score after 36 months was not statistically significant from either 12 months $(\mathrm{p}=0,999)$ or 24 months $(\mathrm{p}=0,924)$. Similarly, for UW-NPS, BPI and SF-36: comparing with scores at 12 months ( $\mathrm{p}=0,999)$ and 24 months $(\mathrm{p}=0,999)$. There were also no statistically significant differences found between any scores at 24 months and at 12 months.

\section{Discussion}

Chronic neuropathic pain is a considerable burden to society with high prevalence of patients refractory to medical treatment. A recent growing interest in its use for chronic pain refractory to medical treatment has paralleled advances in DBS technology, neuroimaging, and the publication of more rigorous case series regarding its long-term efficacy. ${ }^{24}$

Some support from animal evidence derives from Kim et al who developed an experimental trial in rats to observe the effect of DBS in animal models of neuropathic pain. Using the VPL thalamic nucleus as a stimulation target, establishing three groups of animals (normal group, neuropathic pain group and neuropathic pain group treated with DBS) and comparing the behavioural changes, they concluded that DBS was effective in decreasing not only the pain response score but also duration of showing painful response in neuropathic pain. ${ }^{25}$

Two multicenter trials of DBS for pain were conducted to seek US FDA approval, ${ }^{26}$ but neither satisfied efficacy criteria of at least half of the patients reporting at least 50\% pain relief one year after surgery. Thus, US FDA approval was therefore not sought, and DBS for pain was decreed "off label," precluding medical insurance reimbursement. However, in Europe, the European Federation of Neurological Societies (EFNS) and the United Kingdom National Institute for Health and Clinical Excellence (NICE) expressed support for this procedure ${ }^{27,28}$ and consequently, more surgeons currently report DBS for pain inside Europe than in USA.

In this report we describe one recent, open-label, prospective study of DBS for pain, and the largest contemporary single-center experience of DBS for limb injury pain. Our experience with DBS for movement disorders likely benefited from the Oxford neurosurgeons' 15 -year learning curve with DBS for pain, thus supporting an argument for only undertaking DBS for pain in centers with access to appropriate expertise and experience, and willing to audit outcomes. Moreover, the refined patient selection within specific pathologies appeared to have better results than larger heterogeneous case series. ${ }^{29,30,31}$ The results here suggest that DBS can consistently deliver analgesia to patients with chronic neuropathic pain after amputation and brachial plexus injury, with significant sustained improvements 3 years later.

Since the pioneering studies of Mazars et al., Richardson and Akil, and Hosobuchi and Adams, and their long-term follow-up results published in the 1970s and 1980s, ${ }^{19,32,33,34}$ published peer-reviewed clinical outcomes data in DBS for pain case series comprising at least 6 patients were recently 
summarized elsewhere, showing that only about 20 groups worldwide have reported long-term efficacy in up to $83 \%$ of patients with follow-ups of up to 6 years, ${ }^{12}$ and only 6 centers, to our knowledge, have

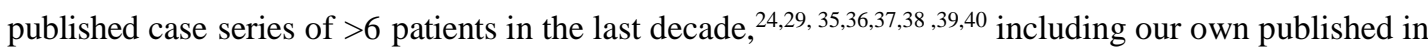
2013. ${ }^{21}$ Two of these centers also reported that DBS can be an effective treatment for various types of intractable head and face pain ${ }^{37,39}$ and one third center for post-stroke intractable neuropathic pain. ${ }^{40}$ However, the other three published large case serious with a heterogeneous selection of patients due to the great variability of etiologies of neuropathic pain, being the limb injury and post-stroke patients were among those groups that showed better results. ${ }^{24,36,38}$

The large variability of results in case series of DBS for pain to date may reflect individual differences between patients as to what constitutes success. Selection of patients represents a real challenge for these studies. ${ }^{12}$ Unlike many others including a great diversity of etiologies of neuropathic pain, in our case series there is a homogeneity across our selected population once they suffered of chronic neuropathic pain after traumatic limb injury, allowing a better outcome assessment. Central neuropathic mechanisms may differ between brachial plexus injury and amputation, but both are forms of traumatic limb injury and we chose to select these patients in Porto while drawing upon the experience in Oxford of success with those particular patient groups. ${ }^{24}$

Besides a mix of heterogeneous cases, other shortcomings of other case series have not affected this study, namely variable deep brain sites stimulated and stimulation parameters, underspecified patient selection criteria and unblinded assessment of patient self-reported outcomes. Moreover, we assured that all patients that underwent this procedure showed improvement in pain scores during the 48 hours postoperative period of clinical assessment, and a decision was made to permanently implant the electrodes. Due to lack of efficacy, despite intraoperative paresthesia, only one brachial plexus injury patient underwent an unsuccessful postoperative trial of externalized DBS and the procedure was aborted.

Our single-center case series shows benefit at three years from VPL-DBS, suggesting that this therapy retains a role in these difficult-to-treat conditions. Regarding quality of life, an intriguing observation from the results presented here, is the considerable and significant improvement after 36 months in pain scores (VAS and BPI, but not UWNPS, as after 12 months), without an important overall improvement in quality of life as measured by SF-36. Nonetheless, meaningful and sustained improvements were noted in physical functioning, social functioning and bodily pain, with negligible improvements in physical role, mental health and emotional role. It is difficult to explain these results without undertaking further qualitative research by interviewing the patients, caregivers, and blinded pain physicians who undertook assessments. In our opinion, this can be due not only to the motor deficit but also to its psychological sequelae, both limiting daily routine despite pain improvement. These findings about SF-36 subscores after 36 months are similar to the ones found in our previous paper after 12 months, with the exception of social functioning. While after 12 months social functioning was negligibly improved, maybe because pain relief on a functionless limb may have contributed little to altering daily activities, now, after 36 months, with a significant improvement in this subscore, one can postulate that patients finally could adapt to daily routine activities, despite a functionless limb, gradual process of adaptation only being reported with a longer follow-up. However, further qualitative, social, and psychological research is desirable to analyse this unexpected finding. 
Other authors are reporting treatments besides DBS, like Motor Cortex Stimulation (MCS), Spinal Cord Stimulation (SCS) and Dorsal Root Entry Zone (DREZ) lesioning. No trials were yet performed comparing the efficacy between these treatments for neuropathic pain, but some case series have been described. In this series, patients were offered DBS as their primary surgical treatment.

A small comparative case series of 19 amputees, followed by a period ranged from 2 to 18 years, suggested that DBS ameliorated phantom limb pain with greater efficacy than MCS (60\% against 20\%) and SCS (60\% against 32\%) ${ }^{41}$ MCS may be considered a more reasonable treatment of chronic intractable neuropathic pain, as it is perceived contentiously by some to be less invasive compared with DBS, despite no statistical differences were found between MCS and DBS group. ${ }^{42}$ However, we consider that this study has a lot of limitations, one of them being the heterogeneity of the selected patients. Sanchs et al, with a cohort of 14 heterogeneous patients reported that MCS failed to produce acceptable long-term benefit. ${ }^{43} \mathrm{MCS}$ has also been undertaken with equivocal results regarding its influence upon paroxysms more than continuous pain. ${ }^{44,45}$ However, improvements in phantom limb pain with DBS have been reported by multiple DBS practitioners. ${ }^{33,34,46,47,48}$ We postulate that in patients with brachial plexus injury or phantom limb pain after amputation, there may be a smaller cortical representation of the limbs, and this was the reason of our choice.

Concerning comparisons between DBS and SCS few published data exist. Two retrospective studies from the same group have compared all 3 modalities of central neurostimulation, but the results are obfuscated first by different treatments trialled, both between and sequentially within patients, and second by limited outcome information. ${ }^{41,49}$ Recent reviews attempting to compare these three procedures have been limited by variable outcome measures and a heterogeneous case mix. ${ }^{50,51}$

Brachial plexus injury pain has a continuous component, often described as burning or throbbing, and a paroxysmal one. The existence of several classification schemes for these lesions reflect the variety of mechanisms and established lesions and, at least in part, can be related to the diversity of pain patterns. Historically, DREZ selective lesioning through microsurgical incision (Sindou technique) or with radiofrequency thermocoagulation (Nashold technique) is particulary effective for the treatment of the paroxysmal component of the pain. ${ }^{52}$ DREZ has shown greatest improvements in paroxysmal pain; two-thirds of patients improved long term, yet as many as $10 \%$ experience dysesthesia and ipsilateral leg weakness. ${ }^{53,54,55}$ DREZ ultimately is a lesioning technique with a significant percentage recurrence of pain, as reported elsewhere..$^{56,57}$

Several case series suggest that at least $25 \%$ of patients do not experience long-term success beyond one year after DBS for pain when they were successful during trial stimulation, ${ }^{24,58}$ and progressive increases of stimulus amplitude have proven unhelpful. ${ }^{48}$ Tolerance can be overcome by subtle alterations of either pulse width, frequency, or both, or failing that, breaks in stimulation. ${ }^{24}$ The overall results after 36 months suggest that our patients are still experiencing long-term benefit from this procedure. Nevertheless, the stimulation parameters were changed and adapted. Initial mean parameters were an amplitude of $2.1 \pm 1.1 \mathrm{~V}$, a frequency of $23.3 \pm 13.5 \mathrm{~Hz}$ and a pulse of $186.7 \pm 76.5 \mu \mathrm{s}$. After 36 months, all the parameters had increased: $4.1 \pm 0.9 \mathrm{~V}, 15.3 \pm 6.7 \mathrm{~Hz}$ and $196 \pm 51.8 \mu \mathrm{s}$. 
No statistically significant differences were found between 1,2 and 3 year time periods, supporting the idea that DBS retained its efficacy. However, long-term follow-up of the patients in this cohort beyond three years will be important to assess adequately for tolerance phenomena.

Limitations to this study include the small case series size, there was neither randomization nor control comparable group. DBS can be reversed, with the patient blinded to device status, enable the same patient to be tested both on and off DBS in contiguous randomly ordered paired of trials. This so-called "N-of-1" trial paradigm was developed to assess the efficacy of analgesic treatments and seems ideal to assessing DBS for pain. ${ }^{59}$ Aside from needed RCT issues of funding, recruitment, and follow-up, the power such studies have to detect a difference between groups relies upon setting a meaningful threshold of clinical improvement with a ratified outcome score. Historically, 50\% improvement in a total pain score has been used, but such sustained quantitative improvements are unusual in a population of patients whose chronic pain waxes and wanes, and may be ameliorated by therapy in some qualitative components of pain more than others. Alternative thresholds and approaches to study design are therefore desirable alongside the holy grail of an objectively measurable biomarker for chronic pain.

Neuromodulation for neuropathic pain continues to be studied, with some promising results and positive experiences among physicians, giving hope to patients with this difficult-to-treat pain and raising their expectations and quality of life. Long-term follow-up of an intensively studied cohort contributes to this evidence, yet few randomized, controlled, clinical trials have been performed. ${ }^{50,51}$ Thus, continued randomized, double-blinded, controlled clinical trials evaluating DBS both on and off are needed, as are revisiting comparisons with other neuromodulatory therapies such as MCS and SCS, as a treatment for neuropathic pain after limb injury. 


\section{References}

[1] Ashburn MA, Staats PS. Management of chronic pain. Lancet 1999, 353:1865-9;

[2] Kreitler S, Niv D. Cognitive impairment in chronic pain. Pain Clinical Updates 2007, 15:1-4;

[3] Elliott AM, Smith BH, Penny KI, Smith WC, Chambers WA. The epidemiology of chronic pain in the community. Lancet 1999, 354:1248-1252;

[4] Gureje O, Von Korff M, Simon GE, Gater R. Persistent pain and well-being: a World Health Organization Study in Primary Care. JAMA 1998, 280:147-151;

[5] Wilkinson HA, Davidson KM, Davidson RI. Bilateral anterior cingulotomy for chronic noncancer pain. Neurosurgery 1999, 45:1129-1136;

[6] Jensen TS, Baron R, Haanpaa M, et al. A new definition of neuropathic pain. Pain 2011;152:2204-2205;

[7] Torrance N, Smith BH, Bennett MI, Lee AJ. The epidemiology of chronic pain of predominantly neuropathic origin. Results from a general population survey. J Pain 2006;7:281-289;

[8] Papuć E, Rejdak K. The role of neurostimulation in the treatment of neuropathic pain. Ann Agric Environ Med 2013, 1:14-7;

[9] Rokyta R, Fricová J. Neurostimulation methods in the treatment of chronic pain. Physiol Res. 2012, 61 Suppl 2:S2331 ;

[10] Nizard J, Raoul S, Nguyen JP, Lefaucheur JP. Invasive stimulation therapies for the treatment of refractory pain. Discov Med. 2012, 14(77):237-46;

[11] Hall GC, Carroll D, Parry D, McQuay HJ. Epidemiology and treatment of neuropathic pain: the UK primary care perspective. Pain 2006, 122:156-162;

[12] Pereira EA, Aziz TZ. Neuropathic Pain and Deep Brain Stimulation. Neurotherapeutics. 2014, 11(3): 496-507;

[13] Narakas A. Surgical treatment of traction injuries of the brachial plexus. Clin Orthop Relat Res 1978, 133:71-90;

[14] Parry CB. Pain in avulsion of the brachial plexus. Neurosurgery 1984, 15:960-965;

[15] Parry CB. Pain in avulsion lesions of the brachial plexus. Pain 1980, 9:41-53;

[16] Pereira EA, Green AL, Nandi D, Aziz TZ. Deep brain stimulation: indications and evidence. Expert Rev Med Devices 2007, 4:591-603;

[17] Heath R. Studies in Schizophrenia: A Multidisciplinary Approach to Mind-Brain Relationships. Cambridge, MA: Harvard University Press, 1954; 
[18] Hosobuchi Y, Adams JE, Rutkin B. Chronic thalamic stimulation for the control of facial anesthesia dolorosa. Arch Neurol 1973, 29:158-161;

[19] Richardson DE, Akil H. Long term results of periventricular gray self-stimulation. Neurosurgery 1977, 1:199202 ;

[20] Mark VH, Ervin FR. Role of thalamotomy in treatment of chronic severe pain. Postgrad Med 1965, 37:563-571;

[21] Pereira EA, Boccard SG, Linhares P, et al. Thalamic deep brain stimulation relieves neuropathic pain after amputation or brachial plexus avulsion. Neurosurg Focus 2013;35:1-11;

[22] Cleeland CS, Ryan KM. Pain assessment: global use of the Brief Pain Inventory. Ann Acad Med Singapore 1994, 23:129-138;

[23] Galer BS, Jensen MP. Development and preliminary validation of a pain measure specific to neuropathic pain: the Neuropathic Pain Scale. Neurology 1997, 48:332-338;

[24] Boccard SG, Pereira EA, Moir L, Aziz TZ, Green AL. Long-term outcomes of deep brain stimulation for neuropathic pain. Neurosurgery 2013, 72:221-231;

[25] Kim, J, Lee, SE, et al. The Neuromodulation of Neuropathic Pain by Measuring Pain Response Rate and Pain Response Duration in Animal. J Korean Neurosurg Soc. 2015; 57(1): 6-11;

[26] Coffey RJ. Deep brain stimulation for chronic pain: results of two multicenter trials and a structured review. Pain Med 2001, 2: 183-192;

[27] Cruccu G, Aziz TZ, Garcia-Larrea L, Hansson P, Jensen TS, Lefaucheur JP, et al. EFNS guidelines on neurostimulation therapy for neuropathic pain. Eur J Neurol 2007, 14:952-970;

[28] National Institute for Health and Care Excellence: IPG382. Deep Brain Stimulation for Chronic Pain Syndromes (Excluding Headache). (http://publications.nice.org.uk/deep-brain-stimulation-for-refractory-chronic-pain syndromes-excluding-headache-ipg382). [Accessed July 25, 2013]

[29] Levy RM. Deep brain stimulation for the treatment of intractable pain. Neurosurg Clin N Am 2003, 14:389-399;

[30] Pereira EAC, Aziz TZ. Deep brain stimulation, in Hayek S, Levy RM, Deer TR (eds): Neurostimulation for the Treatment of Chronic Pain. Interventional and Neuromodulatory Techniques for Pain Management, Vol 1. Philadelphia: Elsevier Saunders, 2011, pp 187-201;

[31] Pereira EAC, Moir L, Green AL, Aziz TZ. Deep brain stimulation for chronic pain, in Krames E, Peckham PH, Rezai AR (eds): Neuromodulation, Vol 1. London: Elsevier, 2009, pp 499-507;

[32] Hosobuchi Y, Adams JE, Linchitz R. Pain relief by electrical stimulation of the central gray matter in humans and its rever-sal by naloxone. Science 1977, 197:183-186; 
[33] Levy RM, Lamb S, Adams JE. Treatment of chronic pain by deep brain stimulation: long term follow-up and review of the literature. Neurosurgery 1987, 21:885-893;

[34] Mazars GJ, Merienne L, Cioloca C. Comparative study of electrical stimulation of posterior thalamic nuclei, periaqueductal gray, and other midline mesencephalic structures in man, in Bonica JJ, Liebeskind JC, Albe-Fessard DG (eds): Advances in Pain Research and Therapy. New York: RavenPress, 1979, pp 541-546;

[35] Yamamoto T, Katayama Y, Obuchi T, et al. Thalamic sensory relay nucleus stimulation for the treatment of peripheral deafferentation pain. Stereotact Funct Neurosurg 2006;84:180-183;

[36]. Hamani C, Schwalb JM, Rezai AR, Dostrovsky JO, Davis KD, Lozano AM. Deep brain stimulation for chronic neuropathic pain: Long-term outcome and the incidence of insertional effect. Pain 2006;125:188-196;

[37] Marchand S, Kupers RC, Bushnell MC, Duncan GH. Analgesic and placebo effects of thalamic stimulation. Pain 2003;105:481-488;

[38] Rasche D, Rinaldi PC, Young RF, Tronnier VM. Deep brain stimulation for the treatment of various chronic pain syndromes. Neurosurg Focus 2006;21:E8;

[39] Green AL, Owen SL, Davies P, Moir L, Aziz TZ. Deep brain stimulation for neuropathic cephalalgia. Cephalalgia 2006;26:561-567;

[40] Owen SL, Green AL, Stein JF, Aziz TZ. Deep brain stimulation for the alleviation of post-stroke neuropathic pain. Pain 2006;120:202-206;

[41] Katayama Y, Yamamoto T, Kobayashi K, Kasai M, Oshima H, Fukaya C. Motor cortex stimulation for phantom limb pain: comprehensive therapy with spinal cord and thalamic stimulation. Stereotact Funct Neurosurg 2001, 77:159162 ;

[42] Son, BC, Kim, DR, Kim, H-S, Lee, -W. Simultaneous Trial of deep brain and motor cortex stimulation in chronic intractable neuropathic pain. Stereotact. Funct. Neurosurg. 2014, 92(4):218-26;

[43] Sachs AJ, Babu H, Su YF, Miller KJ, Henderson JM. Lack of efficacy of motor cortex stimulation for the treatment of neuropathic pain in 14 patients. Neuromodulation.2014 Jun;17(4):303-10;

[44] Ali M, Saitoh Y, Oshino S, Hosomi K, Kishima H, Morris S, et al. Differential efficacy of electric motor cortex stimulation and lesioning of the dorsal root entry zone for continuous vs paroxysmal pain after brachial plexus avulsion. Neurosurgery 2011, 68:1252-1258;

[45] Lefaucheur JP, Drouot X, Cunin P, Bruckert R, Lepetit H, Créange A, et al. Motor cortex stimulation for the treatment of refractory peripheral neuropathic pain. Brain 2009, 132:1463-1471;

[46] Hosobuchi Y. Subcortical electrical stimulation for control of intractable pain in humans. Report of 122 cases (1970-1984). J Neurosurg 1986, 64:543-553; 
[47] Kumar K, Toth C, Nath RK. Deep brain stimulation for intractable pain: a 15-year experience. Neurosurgery 1997 , 40:736-747;

[48] Young RF, Rinaldi PC. Brain stimulation, in North RB, Levy RM (eds): Neurosurgical Management of Pain. New York: Springer 1997, 283-301;

[49] Katayama Y, Yamamoto T, Kobayashi K, Kasai M, Oshima H, Fukaya C. Motor cortex stimulation for post-stroke pain: comparison of spinal cord and thalamic stimulation. Stereotact Funct Neurosurg 2001;77:183-186;

[50] Coffey RJ, Lozano AM. Neurostimulation for chronic noncancer pain: an evaluation of the clinical evidence and recommendations for future trial designs. J Neurosurg 2006;105:175-189;

[51] Levy R, Deer TR, Henderson J. Intracranial neurostimulation for pain control: a review. Pain Physician 2011;13:157-165;

[52] Konrad, P. Dorsal Root Entry Zone lesion, Midline Myelotomy and Anterolateral Cordotomy. Neurosurgical Clinics of North America 25, 2014, 699-722;

[53] Aichaoui F, Mertens P, Sindou M. Dorsal root entry zone lesioning for pain after brachial plexus avulsion: results with special emphasis on differential effects on the paroxysmal versus the continuous components. A prospective study in a 29-patient consecutive series. Pain 2011, 152:1923-1930;

[54] Friedman AH, Nashold BS Jr, Bronec PR. Dorsal root entry zone lesions for the treatment of brachial plexus avulsion inju-ries: a follow-up study. Neurosurgery 1988, 22:369-373;

[55] Sindou MP, Blondet E, Emery E, Mertens P. Microsurgical lesioning in the dorsal root entry zone for pain due to brachial plexus avulsion: a prospective series of 55 patients. J Neurosurg 2005, 102:1018-1028;

[56] Ko AL,Ozpinar A, Raskin JS, Magill ST, Raslan AM, Burchiel KJ. Correlation of preoperative MRI with the long-term outcomes of dorsal root entry zone lesioning for brachial plexus avulsion pain. J Neurosurg 2016; 124(5):1470-8;

[57] Sindou M, Mertens P, Wael M. Microsurgical DREZotomy for pain due to spinal cord and/or cauda equina injuries: long-term results in a series of 44 patients. Pain. 2001; 92(1-2):159-71;

[58] Gybels JM, Sweet WH: Neurosurgical Treatment of Persis-tent Pain. Physiological and Pathological Mechanisms of Human Pain. Basel: Karger, Vol 11, 1989;

[59] Green AL, Shad A, Watson R, Nandi D, Yianni J, Aziz TZ. N-of-1 trials for assessing the efficacy of deep brain stimulation in neuropathic pain. Neuromodulation 2004, 7:76-81 
